\title{
Efeito da mepivacaína $2 \%$ nos parâmetros cardiovasculares em cirurgias de terceiros molares
}

The $2 \%$ mepivacaine in the cardiovascular system in third molar surgeries

Efecto de la mepivacaína $2 \%$ en los parámetros cardiovasculares en cirugías de terceros molares

Herlania Silva FREIRE ${ }^{1}$

Roque Soares MARTINS NETO²

Felipe Evangelista VERISSIMO ${ }^{3}$

Pedro Henrique Acioly Guedes Peixoto VIEIRA ${ }^{4}$

Paulo Goberlânio de Barros SILVA

Andressa Aires ALENCAR ${ }^{6}$

Diego Felipe Silveira ESSES

${ }^{I}$ Pós-graduanda em Gestão em Saúde pela Universidade da Integração Internacional da Lusofonia Afro-Brasileira-UNILAB

${ }^{2}$ Cirurgião Buco-Maxilo-Facial (UFMA), Mestrando em Ciências Odontológicas (ênfase em Cirurgia e Traumatologia-Buco-Maxilo-Facial), Unichristus, Fortaleza-CE ${ }^{3}$ Graduado em Odontologia pelo Centro Universitário Católica de Quixadá-UNICATÓLICA

${ }^{4}$ Doutorando em Odontologia (Clínica Odontológica-Cariologia) pela Universidade Federal do Ceará - UFC, 60430-160 Fortaleza-CE ${ }^{5}$ Doutor em Odontologia (Estomatopatologia) pela Universidade Federal do Ceará-UFC

${ }^{6}$ Residente em Saúde da Família e Comunidade pela Residência Integrada em Saúde da Escola de Saúde Pública do Ceará

${ }^{7}$ Mestre em Odontologia (Cirurgia e Traumatologia Bucomaxilo Facial) pela Universidade Federal do Ceará - UFC

\section{Resumo}

Ao serem administrados, os Anestésicos Locais e Vasoconstritores (VC) iniciam os processos de absorção e eliminação, passando pela circulação sanguínea podendo atingir níveis tóxicos ou induzir alterações cardiovasculares. Objetivou-se avaliar o comportamento da atividade cardiovascular nas cirurgias para a remoção de terceiros molares utilizando a mepivacaína $2 \%$ com adrenalina na concentração de 1:100.000 em um período de até 120 min após a injeção anestésica. Trata-se de um estudo longitudinal, interventivo com caráter quantitativo e descritivo, com dados mensuráveis para análise e interpretação, realizado com pacientes da disciplina de clínica cirúrgica do curso de odontologia da UniCatólica. Os resultados obtidos foram analisados através do teste de normalidade de Kolmogorov-Smirnov e comparados ao longo do trans-operatório pelos testes ANOVA para medidas repetidas seguidas do pós-teste de Bonferroni ou Friedman seguido do pós-teste de Dunn adotando um nível de $5 \%$ de significância (p < 0,05). O procedimento com o anestésico utilizado não provocou alterações na Pressão Arterial Sistólica (PAS) (p=0,712), Pressão Arterial Diastólica (PAD) $(\mathrm{p}=0,098)$ bem como da diferença de PAS e PAD ( $\mathrm{p}=0,546)$. Na Frequência Cardíaca (FC) houve diferença significante nos tempos de 10 min $(\mathrm{p}=0,013)$ e $120 \min (\mathrm{p}=0,013)$. Já na Saturação Periférica $\mathrm{O}_{2}\left(\mathrm{SPO}_{2}\right)$ houve diferença estatística significante nos tempos da $1^{\mathrm{a}}$ visita $(\mathrm{p}=0,001), 0$ $(\mathrm{p}=0,001), 5(\mathrm{p}=0,001)$ e $20(\mathrm{p}=0,001)$. Conclui-se assim, que o anestésico local pode ser utilizado em pacientes normotensivos durante a realização de procedimentos cirúrgicos odontológicos, e este leve aumento na $\mathrm{FC}$ e $\mathrm{SPO}_{2}$ pode estar relacionado à ansiedade e/ou ao estresse emocional dos pacientes.

Descritores: Anestésicos Locais; Mepivacaína; Sistema Cardiovascular; Pressão Arterial.

\section{Abstract}

When injected into the tissues, Local Anesthetics and Vasoconstrictors (VC) initiate the process of absorption and elimination, passing through the bloodstream to reach toxic levels or induce cardiovascular changes. The aim of this study was to evaluate the behavior of cardiovascular activity in the surgeries for the removal of third molars using mepivacaine $2 \%$ with adrenaline at a concentration of 1 : 100,000 in a period of up to 120 min after the anesthetic injection. It is a longitudinal, interventional study with a quantitative and descriptive character, with measurable data for analysis and interpretation, carried out with patients of the discipline of surgical clinic of the dentistry course of the UniCatólica. The results obtained were analyzed using the normality test of Kolmogorov-Smirnov and compared during the trans-operative period by ANOVA for repeated measurements followed by the Bonferroni or Friedman post-test followed by the Dunn post-test adopting a 5\% level of significance (p <0.05). The procedure with the anesthetic used did not cause changes in systolic blood pressure (SBP) $(\mathrm{p}=0.712)$, diastolic blood pressure $(\mathrm{DBP})(\mathrm{p}=0.098)$ as well as SBP and DBP difference $(p=0.546)$. In heart rate $(H R)$ there was a significant difference in the time of $10 \mathrm{~min}(\mathrm{p}=0.013)$ and $120 \mathrm{~min}(\mathrm{p}=0.013)$. In the $\mathrm{O} 2$ Peripheral Saturation (SPO2) there was a statistically significant difference at the time of the first visit $(\mathrm{p}=0.001), 0(\mathrm{p}=0.001), 5(\mathrm{p}=0.001)$ and 20 $(\mathrm{p}=0.001)$. It is concluded that the local anesthetic can be used in normotensive patients during dental surgical procedures, and this slight increase in HR and SPO2 may be related to the anxiety and / or emotional stress of the patients.

Descriptors: Anesthetics, Local; Mepivacaine; Cardiovascular System; Arterial Pressure.

\section{Resumen}

Al ser administrados, los anestésicos locales y vasoconstrictores (VC) inician los procesos de absorción y eliminación, pasando por la circulación sanguínea pudiendo alcanzar niveles tóxicos o inducir alteraciones cardiovasculares. Se objetivó evaluar el comportamiento de la actividad cardiovascular en las cirugías para la remoción de terceros molares utilizando la mepivacaína $2 \%$ con adrenalina en la concentración de 1: 100.000 en un período de hasta 120 minutos después de la inyección anestésica. Se trata de un estudio longitudinal, interventivo con carácter cuantitativo y descriptivo, con datos mensurables para análisis e interpretación, realizado con pacientes de la disciplina de clínica quirúrgica del curso de odontología de la UniCatólica. Los resultados obtenidos fueron analizados a través del test de normalidad de Kolmogorov-Smirnov y comparados a lo largo del trans-operatorio por las pruebas ANOVA para medidas repetidas seguidas del post-test de Bonferroni o Friedman seguido del post-test de Dunn adoptando un nivel del $5 \%$ de significancia $(\mathrm{p}<0,05)$. El procedimiento con el anestésico utilizado no provocó cambios en la presión arterial sistólica (PAS) $(\mathrm{p}=0,712)$, presión arterial diastólica $(\mathrm{PAD})(\mathrm{p}=0,098)$, así como de la diferencia de PAS y PAD (p = 0,546). En la Frecuencia Cardiaca (FC) hubo diferencia significante en los tiempos de 10 min $(p=0,013)$ y 120 min $(p=0,013)$. En la Saturación Periférica O2 (SPO2) hubo diferencia estadística significante en los tiempos de la $1^{\mathrm{a}}$ visita $(\mathrm{p}=0,001), 0(\mathrm{p}=0,001), 5(\mathrm{p}=0,001)$ y 20 (p = 0,001). Se concluye así que el anestésico local puede ser utilizado en pacientes normotensivos durante la realización de procedimientos quirúrgicos odontológicos, y este leve aumento en la FC y SPO2 puede estar relacionado a la ansiedad y / o al estrés emocional de los pacientes.

Descriptores: Anestésicos Locales; Mepivacaína; Sistema Cardiovascular; Presión Arterial.

\section{INTRODUÇÃO}

Os Anestésicos Locais (AL) são fármacos de uso frequente em Odontologia. $\mathrm{AL}$ atuam no bloqueio de canais de sódio, alterando a sensibilidade local de uma determinada área do corpo ${ }^{1}$. Em seu efeito, podem distribuir-se causando riscos em tecidos excitáveis, tais: Sistema Nervoso Central (SNC) e Sistema Cardiovascular (SCV). Os Vasoconstrictores (VC) são constituintes adicionais aos AL, reduzindo riscos e prologando seus efeitos anestésicos locais ${ }^{2}$. 
A mepivacaína é um anestésico de duração intermediária, sendo uma amida, metabolizada no fígado e possui excreção renal. Cerca de $1 \%$ a $16 \%$ da dose aplicada é excretada pelos rins ${ }^{3,4}$. A dose máxima é de $6,6 \mathrm{mg} / \mathrm{kg}$, não devendo ultrapassar $400 \mathrm{mg}$ ou 11 tubetes anestésicos. A concentração odontológica eficaz é de $2 \%$ (com vasoconstritor) e de 3\% (sem vasoconstritor). Uma de suas vantagens é que esta substância consegue ter um tempo maior de anestesia do que os outros anestésicos sem o uso do vasoconstrictor. É condensada apenas por laboratórios especializados em artigos odontológicos ${ }^{3,5,6}$.

As catecolaminas são usualmente utilizadas como VC, principalmente a adrenalina ${ }^{3}$. Sua atividade farmacológica é potente e supera a dos demais vasoconstritores sobre o Sistema Cardiovascular (SCV) e encontra-se comercialmente disponível nas concentrações de 1:50.000, 1:100.000 ou 1:200.000 nas soluções anestésicas locais ${ }^{1}$.

Há uma dificuldade em estabelecer se as alterações observadas nos parâmetros cardiovasculares, como a PA, têm como razão relevante o aumento de catecolaminas endógenas circulantes, liberadas em uma situação de estresse ${ }^{7}$. A compreensão do grau de ansiedade do paciente constitui ferramenta de grande importância a ser considerada, visto que o estado emocional do indivíduo pode alterar as repercussões hemodinâmicas e respiratórias ${ }^{8,9}$.

Em cirurgias de terceiros molares, a mepivacaína é um anestésico utilizado com frequência, torna-se importante investigar a atividade cardiovascular sob a utilização deste anestésico, associado a um controle da Pressão Arterial (PA) e as manifestações clínicas que possam vir a acontecer.

Assim, o objetivo deste trabalho foi avaliar o efeito da mepivacaína 2\% com adrenalina na concentração de 1:100.000 na pressão arterial (PA), frequência cardíaca ( $\mathrm{FC}$ ) e saturação periférica de $\mathrm{O}_{2}$ $\left(\mathrm{SPO}_{2}\right)$ em cirurgias ambulatoriais para a remoção de terceiros molares.

\section{MATERIAL E MÉTODO}

O presente estudo foi aprovado pelo Comitê de Ética e Pesquisa (CEP) do Centro Universitário Católica de Quixadá - UniCatólica com protocolo de número 2.013.969 atendendo aos termos da resolução 466/12 do Conselho Nacional de Saúde. Trata-se de um estudo do tipo longitudinal, realizado entre os meses de fevereiro a abril do ano 2017, clínico interventivo com caráter quantitativo e descritivo, com dados mensuráveis para análise e interpretação. Tendo sido realizado na Clínica Escola de Odontologia do Complexo São João Calábria do Centro Universitário Católica de Quixadá. Foram incluídos no estudo pacientes de ambos os sexos, na faixa etária de 18 a 50 anos, dentro das classificações
ASA I e ASA II da Sociedade Americana de Anestesiologistas (ASA) (Quadro 1), e que concordassem em participar da pesquisa assinando o Termo de Consentimento Livre e Esclarecido (TCLE).

Foram excluídos da pesquisa os pacientes que apresentaram desvios do padrão normal dos sinais vitais e dentes com suspeita de alto grau de dificuldade ou risco para a remoção sob anestesia local; pacientes com alterações renais, hepáticas, cardiológicas, glicêmicas ou com desvios nos valores dos exames laboratoriais, ou usando medicamentos que interfiram no metabolismo e na eliminação dos AL, imunossupressores, ou com conhecida história de alergia aos AL.

A coleta de dados foi realizada pelo pesquisador, após aprovação pelo CEP do Centro Universitário Católica de Quixadá - UniCatólica, por meio de questionário de saúde semi-estruturado. Todos os pacientes foram submetidos ao TCLE, explicando, com linguagem apropriada todos os benefícios e possíveis riscos, aos quais, os mesmos estiveram sujeitos no decorrer do estudo. Foi realizada anamnese detalhada onde os pacientes relataram o uso de qualquer medicamento e sua finalidade, para que uma avaliação pudesse ser feita e qualquer risco eventual fosse descartado, e em seguida a coleta dos dados, conforme ficha de avalição com aferição da Pressão Arterial Sanguínea, da Frequência Cardíaca e da Saturação Periférica de $\mathrm{O}_{2}$ nos tempos de $0,3,5,10,15,20,30,40,60,90$ e 120 minutos, totalizando um tempo de 02 (duas) horas de avaliação para análise de possíveis evoluções e intercorrências durante $o$ ato cirúrgico. Foi elaborado um banco de dados, e estabelecido correlações entre as informações coletadas. Utilizouse o programa estatístico Microsoft Excel ${ }^{\circledR} 2013$ para o tratamento dos dados e a análise descritiva foi apropriada aos resultados obtidos e, os resultados foram expostos em tabelas e gráficos.

\begin{tabular}{l} 
Quadro 1 - Critérios de avaliação física \\
\begin{tabular}{|l|l|}
\hline ASA - I & Paciente saudável normal \\
\hline ASA - II & Paciente com doença sistêmica leve e moderada \\
\hline ASA - III & $\begin{array}{l}\text { Paciente com doença sistêmica severa que limita a } \\
\text { atividade, mas não é incapacitante }\end{array}$ \\
\hline ASA - IV & $\begin{array}{l}\text { Paciente com doença sistêmica severa que limita a } \\
\text { atividade e é uma ameaça constante à vida }\end{array}$ \\
\hline ASA - V & $\begin{array}{l}\text { Paciente moribundo, cuja sobrevida esperada é } \\
\text { inferior a 24 horas com ou sem cirurgia. }\end{array}$ \\
\hline ASA - VI & $\begin{array}{l}\text { Paciente clinicamente morto sendo mantido para } \\
\text { transplante de órgãos }\end{array}$ \\
\hline
\end{tabular} \\
\hline
\end{tabular}

Os dados foram exportados do Microsoft Excel para o software GraphPad Prism 5,0 no qual as análises foram realizadas adotando uma confiança de 95\%. Foram expressas as médias e os desvios-padrão das pressões arteriais sistólica, diastólica e diferencial, além da frequência cardíaca e saturação de oxigênio. Estas foram avaliadas pelo teste de normalidade de Kolmogorov-Smirnov e comparadas 
ao longo do trans-operatório pelos testes ANOVA para medidas repetidas, seguido do pós-teste de Bonferroni (dados paramétricos) ou Friedman, seguido do pós-teste de Dunn (dados não paramétricos).

\section{RESULTADOS E DISCUSSÃO}

A amostra foi composta por 19 pacientes, entre 20 e 51 anos, 16 do sexo feminino e 03 do masculino, e a partir de análise obtiveram-se os resultados apresentados na Tabela 1.

Tabela 1 - Médias e desvios-padrão entre Pressões Arteriais Sistólica, Diastólica e Diferencial, a Frequência Cardíaca e a Saturação de Oxigênio dos 19 pacientes enquadrados no estudo

\begin{tabular}{|c|c|c|c|c|c|}
\hline & \multicolumn{3}{|c|}{ Pressão Arterial } & \multirow{2}{*}{$\begin{array}{c}\text { Frequência } \\
\text { Cardíaca }\end{array}$} & \multirow{2}{*}{$\begin{array}{c}\text { Saturação } \\
\mathrm{O}_{2}\end{array}$} \\
\hline & Sistólica & Diastólica & Diferencial & & \\
\hline \multicolumn{6}{|l|}{ Momento } \\
\hline $1^{\text {a }}$ Visita & $133,3 \pm 14,0$ & $88,7 \pm 8,1$ & $44,5 \pm 9,8$ & $88,7 \pm 10,4$ & $93,8 \pm 10,6^{*}$ \\
\hline 0 & $129,7 \pm 21,0$ & $86,8 \pm 13,4$ & $42,8 \pm 14,2$ & $85,8 \pm 12,5$ & $97,0 \pm 10,3 *$ \\
\hline 3 & $130,8 \pm 18,8$ & $87,9 \pm 19,5$ & $42,9 \pm 9,5$ & $89,8 \pm 9,4$ & $96,8 \pm 8,8$ \\
\hline 5 & $129,6 \pm 23,4$ & $84,0 \pm 18,7$ & $45,5 \pm 9,1$ & $88,2 \pm 11,4$ & $97,0 \pm 7,1 *$ \\
\hline 10 & $129,1 \pm 20,1$ & $82,5 \pm 12,6$ & $46,6 \pm 12,5$ & $89,4 \pm 15,8^{*}$ & $95,8 \pm 8,8$ \\
\hline 15 & $131,1 \pm 17,4$ & $87,0 \pm 14,0$ & $44,1 \pm 9,7$ & $85,8 \pm 11,9$ & $96,2 \pm 7,3$ \\
\hline 20 & $130,3 \pm 18,4$ & $86,0 \pm 16,2$ & $44,3 \pm 9,6$ & $85,4 \pm 15,1$ & $97,6 \pm 3,9 *$ \\
\hline 30 & $136,1 \pm 17,4$ & $92,8 \pm 12,9$ & $43,2 \pm 12,3$ & $82,3 \pm 14,9$ & $92,5 \pm 10,7$ \\
\hline 40 & $136,1 \pm 20,6$ & $92,2 \pm 18,1$ & $43,8 \pm 7,2$ & $83,3 \pm 12,6$ & $93,7 \pm 8,5$ \\
\hline 60 & $136,0 \pm 17,9$ & $91,0 \pm 12,8$ & $45,0 \pm 8,9$ & $81,5 \pm 11,3$ & $94,7 \pm 8,5$ \\
\hline 90 & $133,4 \pm 18,7$ & $92,7 \pm 12,5$ & $40,6 \pm 9,6$ & $82,3 \pm 13,9$ & $93,1 \pm 10,4$ \\
\hline 120 & $132,7 \pm 18,8$ & $92,6 \pm 12,5$ & $40,0 \pm 9,5$ & $82,0 \pm 14,4^{*}$ & $93,1 \pm 10,4$ \\
\hline p-Valor & $0,712^{\mathrm{a}}$ & $0,098^{\mathrm{b}}$ & $0,546^{\mathrm{a}}$ & $0,013^{\mathrm{a}}$ & $<0,001^{\mathrm{b}}$ \\
\hline
\end{tabular}

A partir dos dados obtidos da amostra ( $\mathrm{n}=19)$, os parâmetros PAS, PAD e $\Delta \mathrm{S}$ (PAS-PAD), $\mathrm{FC}$ e $\mathrm{SPO}_{2}$ foram avaliados. Nos tempos de $1^{\mathrm{a}}$ visita até 120 minutos, não houve diferença estatística da PAS ( $\mathrm{p}=0,712), \operatorname{PAD}(\mathrm{p}=0,098)$ bem como da diferença de PAS e PAD $(\mathrm{p}=0,546)$. Na FC houve diferença estatisticamente significante nos tempos de 10 minutos $(\mathrm{p}=0,013)$ e 120 minutos $(\mathrm{p}=0,013)$. Já na $\mathrm{SPO}_{2}$ houve diferença estatística significante nos tempos da $1^{\text {a }}$ visita $(\mathrm{p}=0,001), 0 \quad(\mathrm{p}=0,001), 5$ $(\mathrm{p}=0,001)$ e $20(\mathrm{p}=0,001)$ (Tabela 1$)$.

As alterações no sistema cardiovascular relacionadas à utilização de agentes vasoconstritores podem ser influenciadas por vários fatores, como infiltração intravascular, interação do anestésico e/ou vasoconstritor com outras drogas e absorção sistêmica do vasoconstritor. Além disso, a dor causada pela própria injeção e o estresse psicológico do paciente parece estar fortemente relacionada ao aparecimento de alterações no sistema cardiovascular $^{10}$.

Corroborando com o estudo de Dantas et al. ${ }^{11}$, o procedimento de anestesia local (mepivacaína $2 \%$ com adrenalina 1:100.000) não provocou alterações estatisticamente significantes na pressão arterial dos pacientes avaliados, mesmo naqueles em que uma maior quantidade de tubetes de anestésico foi utilizada.

Correlacionando os resultados do segundo parâmetro (FC) avaliado neste estudo com os resultados de Nichols ${ }^{11}$ que monitorizou pacientes normotensos e hipertensos com aferições uma vez a cada minuto durante todo o procedimento cirúrgico e restaurador, atesta-se haver diferenças significativas da FC entre os cinco minutos iniciais e finais do protocolo de atendimento. Porém, em um estudo de coorte, Zottis et al. $^{12}$ avaliaram o efeito de vasoconstritor sobre a PAS, PAD e FC em 68 pacientes, comparando três momentos durante os procedimentos, em sua maioria restauradores, demonstrando em seus resultados que não houveram variações significativas dos níveis pressóricos e de $\mathrm{FC}$, quando foram empregados um ou dois tubetes de anestésico. Eles observaram que, em relação ao tempo, a FC foi mais variável que a PA, e que todos os valores estavam dentro dos limites de normalidade. Isto poderá estar associado a outros fatores que serão discutidos logo mais.

Diversos estudos têm demonstrado não haver alteração nas condições hemodinâmicas de pacientes que receberam anestésico local com vasoconstritor ${ }^{13-15}$. Contudo Dutra ${ }^{16}$ nos mostra que os resultados do terceiro parâmetro avaliado neste estudo $\left(\mathrm{SPO}_{2}\right)$ condizem com os seus, confirmando as alterações significativas obtidas nos dois grupos avaliados.

Em relação ao coeficiente de variação $\mathrm{CV}=\mathrm{S} / \mathrm{x} * 100$ dos parâmetros avaliados, tem-se que a PAS e PAD apresentaram coeficiente máximo no tempo 5 e mínimo na $1^{\text {a }}$ visita, no entanto, a $\Delta \mathrm{S}$ foi máxima no tempo 0 e mínima no 40. A FC apresentou distribuição de coeficiente máximo no tempo 30 e mínimo no tempo 3, enquanto a $\mathrm{SPO}_{2}$ foi máxima no tempo 30 e mínima no 20 (Tabela 2). De maneira semelhante Braga et al. ${ }^{17}$, em análise individual dos casos, mostraram que em 18 (42,9\%) dos 40 pacientes avaliados por ele, pelo menos em algum momento dentre os 60 minutos, ocorreu uma variação acima de $20 \%$ em um dos parâmetros avaliados (dados não mostrados). Entretanto, Prado $^{18}$ não observou marcações de diferenças estatísticamente significativas na evolução das PAS, $\mathrm{PAD}$, variação e FC ao longo das etapas do seu estudo onde foram avaliados 30 pacientes com registros de avaliação completos.

Fatores como idade, condições físicas, psicológicas, sistêmicas, interações medicamentosas, entre outras, poderão predispor ou agravar o quadro de um paciente que esteja sob o uso de $\mathrm{AL}^{3}$, motivo que nos levou a classificar pacientes hígidos dentro das margens de segurança para tais variáveis.

A distribuição de valores para PAS, PAD e $\Delta \mathrm{S}$ segue o padrão de dispersão da figura 1 . Os valores máximos para a PAS foram $160 \mathrm{mmHg}$, PAD $120 \mathrm{mmHg}$ e $\Delta \mathrm{S}$ de $60 \mathrm{mmHg}$. Estes resultados vão ao encontro dos achados de Alemany-Martinez et al. ${ }^{19}$, que observaram alterações desprezíveis da pressão arterial, em pacientes submetidos à remoção 
cirúrgica de terceiros molares inferiores sob anestesia com articaína associada à epinefrina. Essas alterações podem ocorrer principalmente durante a osteotomia, a odontosecção e a avulsão dentária, considerados os momentos de maior estresse do procedimento cirúrgico $^{4,20}$. Os valores mínimos para cada parâmetro estão dentro dos padrões de normalidade de PA (Figura 01) que preconiza a Sociedade Brasileira de Hipertensão ${ }^{21}$ que são os que seguem: valor ótimo de pressão arterial: $<120 \times 80 \mathrm{mmHg}$ (12 por 8) e valor normal de pressão arterial: < 130/85 mmHg.

Tabela 2 - Coeficiente de Variação entre Pressões Arteriais Sistólica, Diastólica e Diferencial, a Frequência Cardíaca e a Saturação Periférica de Oxigênio dos 19 pacientes enquadrados no estudo durante o transcirúrgico

\begin{tabular}{|c|c|c|c|c|c|}
\hline & \multicolumn{3}{|c|}{ Pressão Arterial } & \multirow{2}{*}{$\begin{array}{c}\text { Frequência } \\
\text { Cardíaca }\end{array}$} & \multirow{2}{*}{$\begin{array}{c}\text { Saturação } \\
\mathrm{O}_{2}\end{array}$} \\
\hline & Sistólica & Diastólica & Diferencial & & \\
\hline \multicolumn{6}{|l|}{ Momento } \\
\hline $1^{\text {a }}$ Visita & $10,5 \%$ & $9,1 \%$ & $22,0 \%$ & $11,7 \%$ & $11,3 \%$ \\
\hline 0 & $16,2 \%$ & $15,4 \%$ & $33,2 \%$ & $14,6 \%$ & $10,6 \%$ \\
\hline 3 & $14,4 \%$ & $22,2 \%$ & $22,1 \%$ & $10,5 \%$ & $9,1 \%$ \\
\hline 5 & $18,1 \%$ & $22,3 \%$ & $20,0 \%$ & $12,9 \%$ & $7,3 \%$ \\
\hline 10 & $15,6 \%$ & $15,3 \%$ & $26,8 \%$ & $17,7 \%$ & $9,2 \%$ \\
\hline 15 & $13,3 \%$ & $16,1 \%$ & $22,0 \%$ & $13,9 \%$ & $7,6 \%$ \\
\hline 20 & $14,1 \%$ & $18,8 \%$ & $21,7 \%$ & $17,7 \%$ & $4,0 \%$ \\
\hline 30 & $12,8 \%$ & $13,9 \%$ & $28,5 \%$ & $18,1 \%$ & $11,6 \%$ \\
\hline 40 & $15,1 \%$ & $19,6 \%$ & $16,4 \%$ & $15,1 \%$ & $9,1 \%$ \\
\hline 60 & $13,2 \%$ & $14,1 \%$ & $19,8 \%$ & $13,9 \%$ & $9,0 \%$ \\
\hline 90 & $14,0 \%$ & $13,5 \%$ & $23,6 \%$ & $16,9 \%$ & $11,2 \%$ \\
\hline 120 & $14,2 \%$ & $13,5 \%$ & $23,8 \%$ & $17,6 \%$ & $11,2 \%$ \\
\hline
\end{tabular}

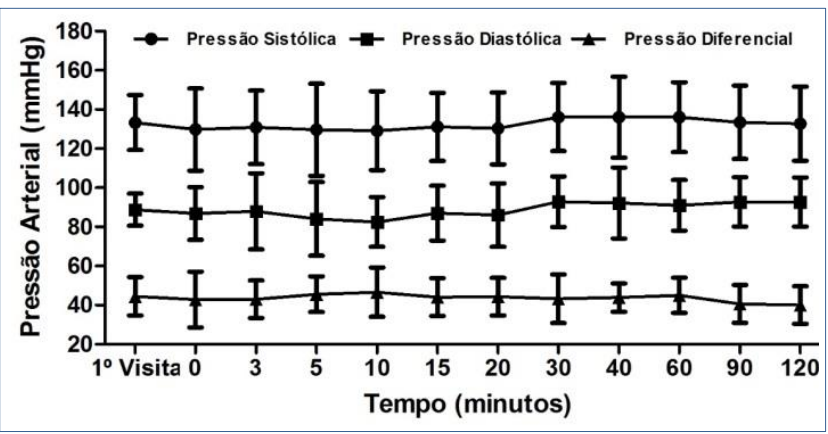

Figura 1: Gráfico de dispersão das Pressões Sistólica, Diastólica e Diferencial (média \pm desvio padrão da média).

Para a FC avaliada em diferentes momentos tem-se que houve diferença estatisticamente significante dos valores nos tempos 10 a 120 minutos (Figura 2), podendo estas alterações ser atribuídas ao vasoconstritor associado ao AL normalmente usado durante a extração de molares, assim como aos outros fatores concomitantes tais como dor, ansiedade ou estresse do paciente ${ }^{11,22-24}$. Alguns autores relataram ausência de repercussões hemodinâmicas em pacientes sob efeitos de anestésico local com epinefrina, ao contrário do observado por outros que encontraram alterações da FC e da PA, sem, no entanto, relacioná-las ao vasoconstritor ${ }^{25,26}$.

$\mathrm{O}$ grau de $\mathrm{SPO}_{2}$ em relação ao tempo apresentou comportamento distinto, porém dentro dos padrões de normalidade. Houve diferença estatisticamente significante nos tempos $1^{\text {a }}$ visita e 0 minutos, $1^{\text {a }}$ visita e 5 minutos, bem como $1^{\text {a }}$ visita e 20 minutos (Figura 3).

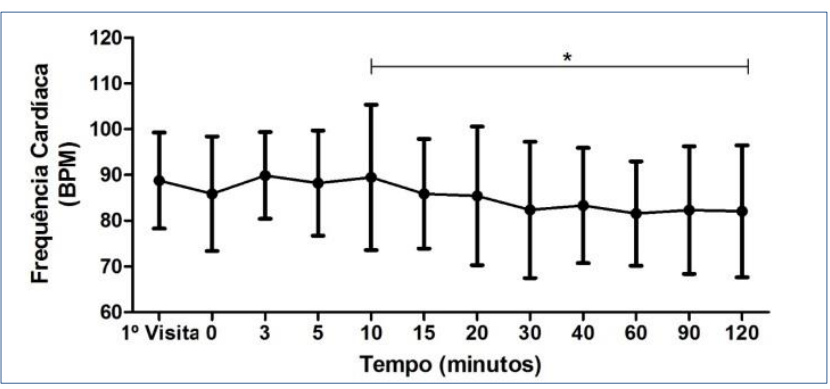

Figura 2: Teste ANOVA para medidas repetidas seguidas do pós-teste de Bonferroni; *p<0,05, média $\pm \mathrm{DP}$.

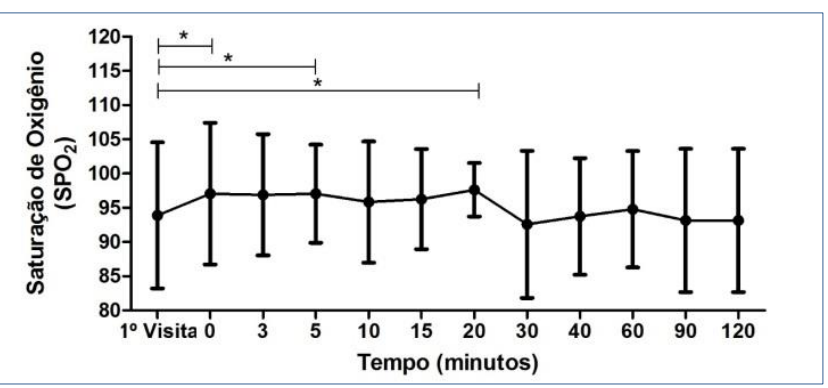

Figura 3: Teste Friedman seguido do pós-teste de Dunn; *p<0,05, média \pm DP.

Concordando com o estudo transversal, prospectivo, descritivo, com componente analítico realizado por Braga et al. ${ }^{17}$ onde foi calculada a diferença na variável dependente $\mathrm{SPO}_{2}$, entre o início da cirurgia e o momento até completar a extração mostrando que os valores médios da $\mathrm{SPO}_{2}$ se mantiveram acima de $95 \%$ (saturação normal) e não apresentaram diferença significativa em relação ao momento inicial utilizando anestésico local com vasoconstritor. A mesma tese é sustentada por Eyigor et al. $^{27}$, quando em estudo prospectivo, randômico e duplo-cego avaliaram 25 pacientes intelectualmente deficientes, estado físico ASA I-II, submetidos à extração dentária sob anestesia geral em ambulatório administrando uma combinação de $2 \mathrm{~g}$ $\mathrm{kg}^{-1}$ de remifentanil e $1 \mathrm{mg} \mathrm{kg}^{-1}$ de lidocaína atestando que nenhum paciente apresentou bradicardia clinicamente significante, hipotensão, rigidez ou hipoxemia.

Algo dito como uma limitação intrínseca à realização deste estudo seria o método de aferição dos parâmetros base. Método este que foi realizado com aparelhos automáticos. Porém, em confronto com a literatura, Pavan et al. ${ }^{28}$ afirmam que valores pressóricos obtidos através do método automático digital apresentam boa concordância com os valores aferidos pelo método convencional auscultatório. Outra limitação foi a amostra, dita por conveniência, que não se adequa a necessidade de resultados mais relevantes, bem como o estado psicológico dos pacientes que poderá ter interferido nos resultados obtidos, já que este fator não foi previamente avaliado. 


\section{CONCLUSÃO}

A utilização do anestésico mepivacaína $2 \%$ com adrenalina (1:100.000) não provocou alterações significantes na PAS e PAD, podendo ser seguramente utilizado em pacientes normotensivos. Já na $\mathrm{FC}$ e $\mathrm{SPO}_{2}$ houve discretas alterações que podem estar relacionadas aos fatores emocionais considerando o estado psicológico do paciente dentro do procedimento que é tido como invasivo por se tratar de uma cirurgia.

Sugere-se a realização de novos estudos utilizando este mesmo anestésico, porém com uma amostra de tamanho maior para que se obtenham resultados mais precisos e fidedignos com o intuito de complementá-lo.

\section{REFERÊNCIAS}

1. Andrade ED. Terapêutica Medicamentosa em Odontologia. 3. ed. São Paulo: Artes Médicas; 2014.

2. Teixeira RN. Anestesia Local sem Vasoconstritor versus com Vasoconstritor. Porto: Faculdade de Ciências da Saúde; 2014.

3. Malamed SF. Manual de Anestesia Local. 5a. ed. São Paulo: Elsevier; 2004.

4. Silvestre FJ, Verdu MJ, Sanchis JM, Grau D, Penarrocha M. Effects of vasoconstrictors in dentistry upon systolic and diastolic arterial pressure. Medicina Oral. 2001;6(1):57-63.

5. Ponzoni D, Sanches MG, Okamoto T. Influência de solução anestésica local contendo mepivacaína no processo de reparo em feridas de extração dental: análise histológica em ratos. Rev ABO. 2003;11(5):287-92.

6. Dicionário de Especialidades Farmacêuticas, 2004/05. 33. ed. Rio de Janeiro: Editora de Publicações Científicas; 2004.

7. Frabett IL, Checchi L, Finelli K. Cardiovascular effects of local anesthesia with epinephrine in periodontal treatment. Quintessence Int. 1992;23(1):19-24.

8. Lambrecth JT, Filippi A. Arrigoni J, Cardiovascular monitoring and its consequences in oral surgery. Ann Maxillofac Surg. 2011;1(2):102-6.

9. Fukayama H, Yagiela J. A. Monitoring of vital signs during dental care. Int Dent $J$. 2006;56(2):102-8.

10.Dantas MVM, Gabrielli MAC, Hochuli-Vieira E. Efeito da mepivacaína 2\% com adrenalina 1:100.000 sobre a pressão sanguínea. Rev Odontol Unesp. 2008;37(3):223-27.

11.Nichols C. Dentistry and hipertension. J Am Dent Assoc. 1997;128(11):1557-62.

12.Zottis D, Bernardes R, Wannmacher L. Efeito de vasoconstritor usado em anestesia local sobre a
PA sistêmica e FC durante o atendimento odontológico. Rev ABO. 1999; 7:289-93.

13.Neves RS, Neves IL, Giorgi DM, Grupi CJ, César LA, Hueb W et al. Effects of epinephrine in local dental anesthesia in patients with coronary artery disease. Arq Bras Cardiol. 2007; 88(5):545-51.

14.Laragnoit AB, Neves RS, Neves IL, Vieira JE. Locoregional anesthesia for dental treatment in cardiac patients: a comparative study of $2 \%$ plain lidocaine and $2 \%$ lidocaine with epinephrine (1:100,000). Clinics. 2009; 64(3):177-82.

15. Silvestre FJ, Salvador-Martínez I, Bautista D, Silvestre-Rangil J. Clinical study of hemodynamic changes during extraction in controlled hypertencontrolled hypertensive patients. Med Oral Patol Oral Cir Bucal. 2011;16(3):e354-58.

16.Dutra RM. Saturação periférica de oxigênio, frequência cardíaca e pressão arterial sistêmica em crianças portadoras de cardiopatia congênita cianogênica com hipofluxo pulmonar durante procedimento odontológico [tese]. São Paulo: Faculdade de Medicina da Universidade de São Paulo/Programa de Pneumologia; 2012.

17. Braga AFA, D'Ottaviano LH, Braga FSS, Morais SS. Extração de terceiros molares retidos sob anestesia local. Avaliação de ansiedade, dor, alterações hemodinâmicas e respiratórias. Rev Fac Odontol Porto Alegre. 2010;51(2):9-14.

18.Prado RMS. Efeitos cardiovasculares da mepivacaína com epinefrina seguida hialuronidase: ensaio duplo-cego controlado em cirurgias bilaterais de terceiros molares inferiores [dissertação]. São Paulo: Faculdade de Odontologia da USP; 2007.

19.Alemany-Martinez A, Valmaseda-Castellón E, Berini-Aytés L, Gay-Escoda C. Hemodynamic changes during the surgical removal of lower third molars. J. Oral Maxillofac. Surg. 2008;66(3):45361.

20.Paramaesvaran M, Kingon AM. Alterations in blood pressure and pulse rate in exodontia patients. Aust Dent J. 1994;39(5):282-86.

21.Sociedade Brasileira de Anestesiologia. Tutorial de Anestesia da Semana - Oximetria de Pulso parte 1. WILSON, Iain. Royal Devon \& Exeter Hospital, UK, 2013. Disponível em: <http://grofsc.net/wp/wp-content/uploads/ 2013/ 03/Oximetria-de-pulso-parte-11.pdf $>$. Acesso em: 22 de novembro, 2016.

22.Tolas AG, Pflug AE, Halter JB. Arterial plasma epinephrine concentrations and hemodynamic responses after dental injection of local anesthetic with epinephrine. J Am Dent Assoc.1982;104(1):41-3.

23.Brand HS, Abraham-Inpijn L. Cardiovascular responses induced by dental treatment. Eur J Oral Sci.1996;104(3):245-52. 
24.Takahashi Y, Nakano M, Kanri KST. The effects of epinephrine in local anesthetics on plasma catecholamine and hemodynamic responses. Odontology.2005;93(1):72-9.

25.Muzyka BC, Glick M. The hypertensive dental patient. J Am Dent Assoc.1997;128(8):1109-20.

26. Báguena JC, Chiva F. Efectos de los anestésicos de uso odontológico sobre la presión arterial y la frecuencia cardíaca. Rev Eur Odontoestomatol. 1999;1(5):291-95.

27.Eyigor C, Cagiran E, Balcioglu T, Uyar M. Comparação dos efeitos de remifentanil e remifentanil + lidocaína em intubação de pacientes intelectualmente deficientes. Rev Bras Anestesiol. 2014;64(4):263-68.

28.Pavan MV, Saura GE, Korkes HÁ, Nascimento KM, Neto NMN, Dávila R, Rodrigues CIS, Almeida FA. Similaridade entre os valores da pressão arterial aferida pelo método auscultatório com aparelho de coluna de mercúrio e o método oscilométrico automático com aparelho digital. J Bras Nefrol. 2012;34(1):43-9.

\section{CONFLITO DE INTERESSES}

Os autores declaram não haver conflitos de interesse.

AUTOR PARA CORRESPONDENCIA

\section{Herlania Silva Freire}

herlaniafreire15@hotmail.com

Submetido em 06/10/2018

Aceito em 08/02/2019 